Pérez-Bonet, G., Velado-Guillén, L.A., García-Domingo, B. \& Sánchez-Fernández, M.L.(2021). Inteligencia emocional y esquemas tempranos desadaptativos en futuros educadores: ampliando fronteras. Revista Electrónica Interuniversitaria de Formación del Profesorado, 24(3), 133-147.

DOI: https://doi.org/10.6018/reifop.435821

\title{
Inteligencia emocional y esquemas tempranos desadaptativos en futuros educadores: ampliando fronteras
}

Gregorio Pérez Bonet ${ }^{(1)}$, Luis Ángel Velado Guillén ${ }^{(1)}$ Begoña García Domingo ${ }^{(2)}$, María Luisa Sánchez Fernández ${ }^{(2)}$

(1) Universidad "Ces Don Bosco", (2) Universidad Complutense de Madrid

\section{Resumen}

El objetivo principal del presente trabajo es llevar a cabo la identificación de los Esquemas Desadaptativos Tempranos en educadores en formación y sus puntuaciones en Inteligencia Emocional Percibida para, a continuación, explorar posibles relaciones entre ellos y establecer diferencias en virtud del género y de la titulación cursada. La muestra es de estudiantes universitarios madrileños de los Grados de Maestro en Educación Infantil, Primaria y Educación Social ( $\mathrm{N}=713$ ), con una media de edad de 18,8 años. Tras la aplicación de la adaptación española del SQ-SF (Cid, Tejero y Torrubia, 1997) y del TMMS-24 (Fernández Berrocal, Extremera y Ramos, 2004), encontramos que los esquemas que alcanzaron mayor puntuación fueron Autosacrificio $(18,28)$, Metas Inalcanzables $(16,45)$ y Abandono $(14,13)$, obteniéndose diferencias significativas en algunos de los esquemas entre las diferentes variables consideradas. Por su parte, los resultados encontrados en Inteligencia Emocional Percibida mostraron puntuaciones medias de 27,22 en Atención a los Sentimientos, de 25,57 en Claridad Emocional y de 26,57 para la Regulación Emocional, también con algunas diferencias significativas entre géneros y titulación. Finalmente se encontraron correlaciones negativas estadísticamente significativas entre la puntuación total de esquemas y las dimensiones de Claridad Emocional y Regulación emocional y positiva con Atención a los sentimientos.

\section{Palabras clave}

Esquemas desadaptativos tempranos; formación; inteligencia emocional; competencia emocional.

\section{Contacto:}

Gregorio Pérez Bonet,goyo@cesdonbosco.com, CES Don Bosco, C/Mª Auxiliadora 9. Este artículo se inscribe en el Proyecto de Investigación "Cognición y emoción en educadores" financiado parcialmente por el Banco Santander. 


\title{
Emotional intelligence and early maladaptive schemas in future educators: expanding borders
}

\begin{abstract}
The main objective of the present work is the identification of the Early Maladaptive Schemes in educators in training and their scores in Perceived Emotional Intelligence (TMMS-24) to then explore possible relationships between them and establish differences by gender and degree taken. The sample is from Madrid university students of the Degrees in Infant, Primary and Social Education ( $\mathrm{N}=713$ ), with an average age of 18.8 years. After the application of the Spanish adaptation of the SQ-SF (Cid, Tejero y Torrubia, 1997) and the TMMS-24 (Fernández Berrocal, Extremera and Ramos, 2004), we found that the schemes that reached highest scores were Self-sacrifice $(18,28)$, Unreachable Goals $(16,45)$ and Abandonment $(14,13)$, obtaining significant differences in some of the schemes between the different levels of the factors considered. On the other hand, the results found in Perceived Emotional Intelligence showed average scores of 27.22 in Attention to Feelings, 25.57 in Emotional Clarity and 26.57 for Emotional Regulation, also with some significant differences between diferent variables. Finally, statistically significant negative correlations were found between the total scheme score and the dimensions of Emotional Clarity and Emotional Regulation, and positive with Attention to Feelings.
\end{abstract}

\section{Key words}

Early maladaptive schemas; training; emotional intelligence ; emotional competence.

\section{Introducción}

Asistimos en la actualidad a una transición dentro de los modelos educativos. Pivotamos de la mera transmisión de conocimientos a un modelo basado en el aprendizaje de competencias (UNESCO, 2018). En este sentido, la adaptación a las numerosas demandas del complejo milenio actual, exige que en la formación de los ciudadanos se incluya el entrenamiento en competencias emocionales (Márquez-Cervantes y Gaeta-González, 2017; Kotsou, et al., 2019).

A pesar de la dificultad de delimitar el constructo "competencias emocionales", podemos considerarlas como un conjunto de conocimientos, capacidades, habilidades y actitudes necesarias para comprender, expresar y regular los fenómenos emocionales (Bisquerra, 2016,p.57).

En esta línea, y además de la inclusión de las competencias emocionales en el curriculum ordinario de la educación obligatoria, se ha sugerido, a partir del conocido Espacio Europeo de Educación Superior (EEES), que dichas competencias se integren al curriculum troncal en la universidad (Cazalla y Molero, 2016; Fernández Berrocal et al., 2017; Puertas Molero et al., 2018). Ello se extiende con mayor interés a los grados de educación (Jiménez Hernández, et al., 2019.).

Debe tenerse en cuenta, además, que el entorno universitario representa un espacio potencialmente idóneo para el desarrollo de estas habilidades al contribuir positivamente a mejorar el bienestar general de los estudiantes (Livia y García, 2015). En este sentido, la 
competencia emocional de los alumnos ha sido analizada en relación con diversas variables (rendimiento académico, capacidad intelectual, género, edad o grado y curso estudiados), sin llegar a alcanzarse resultados concluyentes ni unánimes (Pérez-Bonet y Velado Guillén, 2017; Sanmartín López et al., 2018; entre otros), lo que anima a seguir investigando en este ámbito.

En otro orden de cosas, si la competencia emocional en las escuelas es un objetivo más a alcanzar por nuestros niños y adolescentes, resulta obligado que los futuros docentes estén capacitados para implementar alguna de las muchas actuaciones diseñadas para ayudar a sus alumnos a gestionar, comprender y reconocer sus emociones (Pérez-González, 2012). Para todo ello es necesario que los educadores adquieran un desarrollo emocional adecuado que tendrá, entre otros muchos efectos positivos, un claro impacto sobre su práctica docente y sus relaciones con los estudiantes (Dolev y Leshem, 2017).

Por tanto, si los docentes y futuros docentes tienen que adquirir competencias emocionales, deberían entrenar y mejorar su propia inteligencia emocional, ya que este constructo resulta nuclear en el territorio de las competencias emocionales (Bisquerra, et al., 2015). La pionera conceptualización de la misma, presentada en el modelo de Mayer y Salovey (1997), definía la inteligencia emocional (IE) como una capacidad para expresar y valorar emociones, acceder y generar sentimientos que faciliten el pensamiento, entender la emoción y el crecimiento emocional, así como la capacidad para regular las emociones y promover el crecimiento personal e intelectual.

Entre los beneficios del entrenamiento de la inteligencia emocional para los docentes y futuros docentes se sugieren entre otros: la mejora en las relaciones de apego, la capacidad de trabajar en equipo, incremento de actitudes más empáticas y prosociales (Ortega-Navas, 2014), y en definitiva, una mejora global de su bienestar psicológico (Antoñanzas et al., 2014). Todo ello se traduce en una relación negativa con el síndrome de "burnout" o síndrome del quemado en los profesionales de la educación (Saliari et al., 2011) y con valores superiores en el caso de "engagement", o competencia motivacional positiva vinculada al trabajo (Pena, Rey y Extremera, 2012).

Ampliando perspectivas, la dimensión inteligencia emocional no se puede considerar como un compartimento estanco dentro de la compleja red de factores de personalidad en los educadores. De hecho, la inteligencia emocional se relaciona con la personalidad y ambos con un mejor o peor manejo emocional (Domínguez-Lara, 2018).

Dentro de los posibles constructos vinculados al desarrollo de la personalidad resultan relevantes los Esquemas Desadaptativos Tempranos (EDT), que se han revelado como variables predictivas, entre otras, de inadaptación emocional (Calvete et al., 2013). Los Esquemas Desadaptativos Tempranos se refieren a patrones constituidos por cogniciones, emociones y sensaciones corporales que se reflejan en las relaciones con los demás y con uno mismo (Young et al., 2016). Estos se desarrollan durante la infancia, se elaboran a lo largo de la vida del individuo y son significativamente disfuncionales.

Dichos esquemas se relacionan con diversos desajustes personales, como ansiedad o depresión, con una magnitud moderada en conjunto, según un reciente metaanálisis (Janovsky et al, 2020). También, estos esquemas estarían vinculados con trastornos de personalidad ( Bilge y Balaban 2021).

A pesar de su relevancia para el campo de la personalidad, las investigaciones sobre Esquemas Desadaptativos Tempranos en muestras con profesionales de la educación son muy limitadas. Rittenmyer (1997) encontró asociación entre la escala de "burnout" de Maslach con el exceso en los esquemas de autosacrificio y de metas inalcanzables. En similar 
línea Qahderijani et al. (2015), relacionaron la puntuación total de la escala (EDT) con las puntuaciones de "burnout" en maestros de Educación Primaria. Desde un punto de vista más global, los esquemas desadaptativos se han vinculado de manera negativa con el estado de felicidad percibido en maestros de Educación Física (Gholami et al., 2014). Finalmente, en el estudio de Malahat (2018) se encuentra una correlación negativa entre los esquemas desadaptativos y el grado de responsabilidad en maestros iraníes.

Los estudios que asocian inteligencia emocional y esquemas desadaptativos tempranos son prácticamente inexistentes en la población general. Se ha propuesto, recientemente, que ciertos perfiles emocionales se asociarán con EDT más severos. En este sentido, Tianguan y Joanna (2020) apuntan una correlación de -.660 entre IE y EDT. Estos autores sugieren que una alta inteligencia emocional podría ser un factor protector contra el desarrollo y perpetuación de los EDT.

En definitiva, la investigación sobre Esquemas Desadaptativos Tempranos en docentes es escasa. En absoluto se ha estudiado con muestras españolas de educadores o futuros educadores en formación. Por todo ello, en la presente investigación pretendemos alcanzar los siguientes objetivos:

1.-Describir las puntuaciones tanto de las dimensiones de Inteligencia Emocional Percibida (IEP) como de los Esquemas Desadaptativos Tempranos (EDT) que presentan los estudiantes en una muestra de alumnos de los Grados de Magisterio de Educación Infantil, Educación Primaria y Educación Social.

2.-Identificar diferencias relevantes en los constructos evaluados en virtud de variables sociodemográficas (género y grado).

3.-Explorar la posible asociación entre La inteligencia Emocional y los Esquemas Desadaptativos Tempranos

\section{Metodología}

\section{Diseño y Procedimiento}

Para alcanzar estos objetivos hemos llevado a cabo una investigación empírica cuantitativa que se ajusta a las características de la investigación no experimental, y en la que combinamos estudios asociativos de tipo descriptivo y comparativo. La recogida de datos ha seguido un enfoque transversal selectivo empleando el autoinforme como técnica (Ato et al., 2013).

Los instrumentos para la recogida de datos se aplicaron a lo largo del curso 2019/2020 durante las clases de diversas asignaturas vinculadas al área de Psicología (de forma colectiva y en horario lectivo habitual). El alumnado de primer curso de los Grados de Educación Infantil, Educación Primaria y Educación Social cumplimentó el cuestionario de esquemas (SQ-SF) y el TMMS 24 de inteligencia emocional como parte de las actividades de desarrollo de las competencias de autoconocimiento de dichas asignaturas, de modo que los resultados fueron corregidos y comentados posteriormente en el aula. Los cuestionarios fueron autoadministrados durante una única sesión de clase. Se pidió consentimiento escrito a cada uno de los participantes para la colaboración investigadora.

Para la codificación y análisis de los datos (descriptivo, correlacional y comparativo) se empleó el programa estadístico SPSS versión 22. 


\section{Participantes}

La selección de los sujetos de estudio se llevó a cabo mediante una técnica de muestreo de tipo no probabilístico intencional por conveniencia. La muestra final estuvo formada por 713 estudiantes universitarios de primer curso de las diferentes titulaciones de Grado ofrecidas en el Centro de Enseñanza Superior "Don Bosco" de Madrid (centro adscrito a la UCM) situado en el distrito Moncloa-Aravaca, barrio Ciudad Universitaria. Participaron un total de 310 alumnos del grado de Maestro en Educación Primaria (43,4\%), 282 alumnos del Grado de Maestro en Educación Infantil (39,6\%) y 121 alumnos (17\%) del Grado de Educación Social. La distribución por géneros fue de 143 hombres $(20,1 \%)$ y 570 mujeres $(79,9 \%)$, reflejando la idiosincrasia de los Grados de Educación en la actualidad. La edad media del alumnado de la muestra global fue de 18,8 años.

\section{Instrumentos}

Se ha utilizado el Schema Quiestionnaire-Short Form (SQ-SF, Young \& Brown, 1994) en su adaptación al español por Cid et al., (1997). El cuestionario consta de 75 items y evalúa 15 Esquemas Desadaptativos Tempranos, aglutinados en 5 dominios superiores. El cuestionario ha sido validado por Calvete et al. (2005) para población española. Esta versión corta del cuestionario es más rápida de cumplimentar que la versión posterior, y por ello la preferimos para un primer acercamiento. La escala para evaluar se hace a través de 6 puntos, tipo Lickert: $1=$ Totalmente falso, hasta $6=$ Me describe perfectamente.

Los ítems del cuestionario reflejan los cinco dominios principales de la siguiente forma (Young et al., 2016). Añadimos el a de Cronbach según los autores.

1. Desconexión y Rechazo. Esta dimensión pivota sobre la creencia de que las necesidades propias no serán satisfechas de forma adecuada. Comprende los siguientes esquemas: Deprivación emocional ( $\alpha$ de Cronbach de .84), abandono ( $\alpha$ de Cronbach .84 ) desconfianza ( $a$ de Cronbach .76), defectuosidad ( $a$ de Cronbach .75) y asilamiento social ( $a$ de Cronbach .76)

2. Desempeño y Autonomía deficientes. Este dominio trata sobre las creencias acerca de las dificultades de tener éxito de forma independiente. En este dominio se encontrarían los siguientes esquemas: Vulnerabilidad al daño ( $a$ de Cronbach .66), Apego inmaduro ( $a$ de Cronbach .61), fracaso ( $a$ de Cronbach .85) y dependencia ( $a$ de Cronbach .61)

3. Límites Insuficientes. Este dominio se enfoca en la falta en los límites internos y responsabilidad, tanto hacia las demás personas como hacia metas propias. Los esquemas pertenecientes a este dominio: grandiosidad ( $a$ de cronbach .63) e insuficiente autocontrol ( $a$ de cronbach de.70)

4. Tendencia hacia el otro. Este dominio está orientado hacia los otros. Se presenta una atención excesiva a los demás, a expensas de los propios. A este dominio pertenecen los siguientes esquemas: subyugación ( $\alpha$ de cronbach .68) y autosacrificio ( $\alpha$ de cronbach .77)

- Sobrevigilancia e Inhibición. Este dominio se refiere a la supresión de sentimientos. A este dominio pertenecen los siguientes esquemas: Inhibición emocional (a de cronbach .79) y metas inalcanzables ( $\underline{a}$ de cronbach .69) 
Para la recogida de datos sociodemográficos se incluyeron las siguientes variables: Grado, género, y edad.

Para la evaluación de la Inteligencia Emocional Percibida se ha utilizado el TMMS-24 (Fernández Berrocal et al., 2004), adaptación española del TMMS-48 (Salovey et al., 1995). El TMMS-24 está compuesto de 24 items que se puntúan a través de una escala Likert de 5 puntos, comprendiendo el rango de opiniones entre "Nada de acuerdo" y "Totalmente de acuerdo". Los 24 reactivos se distribuyen en tres constructos, con 8 items cada uno:

1. Percepción o Atención Emocional (AE): medida en que prestamos atención a nuestros estados de ánimo o sentimientos o pensamos sobre ellos (a de cronbach 0,9 según atores)

2. Comprensión o Claridad de Sentimientos (CS): grado en que comprendemos apropiadamente nuestros estados emocionales (a de cronbach de 0,86 según autores)

3. Regulación o Reparación emocional (RE): nivel de habilidad de autorregulación percibida con relación a nuestros estaos emocionales ( $a$ de cronbach de 0,86 según autores).

\section{Resultados}

Para una mejor comprensión, presentamos los resultados obtenidos de forma separada para cada uno de los objetivos propuestos.

1. Describir las puntuaciones tanto de las dimensiones de Inteligencia Emocional Percibida (IEP) como de los Esquemas Desadaptativos Tempranos (EDT) que presentan los estudiantes en una muestra de alumnos de los Grados de Magisterio de Educación Infantil, Educación Primaria y Educación Social.

Se realizó en primer lugar un análisis descriptivo de los quince factores de primer orden del Cuestionario de Esquemas de Young (SQ-SF) y de las 3 dimensiones constitutivas de Inteligencia Emocional Percibida (TMMS-24).

De cara al análisis de los efectos del género y grado cursado sobre las variables sometidas a medida, llevamos a cabo la comprobación del supuesto de normalidad exigible para el uso de pruebas de contraste paramétricas. En este sentido, se sometió a los datos a pruebas de bondad de ajuste (Kolmogorov-Smirnov para una muestra a un nivel de significación de 0,05), encontrándose que ninguno de los esquemas se distribuía según un modelo normal $(p<0.05)$. Por todo ello, las posteriores comparaciones entre grupos se efectuaron empleando pruebas de contraste no paramétricas para muestras independientes, también a un nivel de confianza del $95 \%$.

Sin embargo, y respecto a los factores vinculados con la inteligencia emocional percibida (IEP), sí existió ajuste a una distribución dentro de los límites de la normalidad, por lo que se emplearon pruebas paramétricas.

La tabla 1 recoge los estadísticos descriptivos obtenidos en todas las variables sometidas a medida (media aritmética, desviación típica e indicadores de asimetría y curtosis y a de Cronbach), tanto para la muestra total como diferenciada por género. Como puede verse en ella, los esquemas más activados para la muestra total serían por este orden: autosacrificio (media=18,28), metas inalcanzables (media=16,45) y abandono (media=14,13). De igual 
modo, y también en el mismo orden, son los esquemas más activados tanto en la muestra de mujeres como en la de los hombres.

Tabla 1

Descriptivos básicos por género

\begin{tabular}{cccccccc}
\hline Esquemas & Media & D.T. & Asimetría & Curtosis & Varón & Mujer & $a$ \\
\hline Privación(Pe) & 7,64 & 4,03 & 2,34 & 6,05 & 7,86 & 7,58 & .84 \\
Abandono (Ab) & 14,13 & 5,84 & .45 & $-0,48$ & 13,09 & 14,39 & .83 \\
Desconfianza (De) & 11,34 & 4,80 & .99 & .90 & 10,57 & 11,54 & .79 \\
Aislamiento (Ai) & 8,79 & 4,14 & 1,81 & 3,77 & 8,78 & 8,80 & .77 \\
Imperfección(Im) & 7,21 & 3,20 & 2,75 & 10,43 & 6,91 & 7,28 & .76 \\
Fracaso (Fr) & 8,68 & 4,10 & 1,82 & 4,44 & 8,62 & 8,70 & .86 \\
Dependencia (Dp) & 7,83 & 3,07 & 1,61 & 3,67 & 7,69 & 7,86 & .60 \\
Vulnerabilidad(Vp) & 11,57 & 4,90 & .82 & .28 & 10,31 & 11,89 & .72 \\
Apego inmaduro (Ap) & 10,00 & 4,35 & 1,12 & 1,04 & 9,20 & 10,20 & .68 \\
Subyugación (Su) & 9,32 & 3,92 & 1,36 & 2,03 & 9,06 & 9,38 & .73 \\
Autosacrificio (Au) & 18,28 & 5,60 & .08 & -.74 & 16,76 & 18,66 & .83 \\
Inhibición emocional (le) & 10,85 & 5,50 & 1,10 & .59 & 11,10 & 10,78 & .84 \\
Insuficiente Autocontrol (Ia) & 11,93 & 4,78 & .86 & .73 & 12,62 & 11,76 & .76 \\
Grandiosidad(Gr) & 13,17 & 4,82 & .40 & -.27 & 13,64 & 13,05 & .69 \\
Metas inalcanzables (Mi) & 16,45 & 5,09 & .13 & -.44 & 16,34 & 16,48 & .73 \\
TOTAL ESQUEMAS & 167,20 & 37,8 & .77 & .92 & 162,54 & 168,37 & .92 \\
Atención sentimientos & 27,22 & 6,17 & .09 & -043 & 25,48 & 27,66 & .90 \\
Claridad emocional & 25,57 & 6,37 & .09 & -.41 & 26,43 & 25,36 & .92 \\
Regulación emocional & 26,57 & 6,31 & -.02 & -.05 & 27,59 & 26,31 & .89 \\
\hline
\end{tabular}

Fuente: Elaboración propia

Aunque en la mayoría de los casos parece que encontramos mayor activación en los esquemas en las mujeres que en los hombres, existen algunas excepciones en los que el patrón se invierte como en el caso de los esquemas de grandiosidad, insuficiente autocontrol, inhibición emocional y privación, que obtienen medias aritméticas superiores a las de las mujeres. Respecto a la fiabilidad de las variables medidas a partir del a de Cronbach, todas las variables se encuentran en rangos adecuados a excepción del esquema de dependencia (.60). 


\section{2.- Identificar diferencias relevantes en los constructos evaluados en virtud de variables sociodemográficas (género y la titulación cursada).}

En cuanto a las posibles diferencias en función del género de los estudiantes de la muestra, las pruebas de contraste estadístico efectuadas (Tabla 2) mostraron que las puntuaciones en el esquema de abandono son estadísticamente superiores en las mujeres $(U=45,95 ; p=.017)$, aunque su tamaño efecto es prácticamente inexistente ( $r=.09)$. El esquema de vulnerabilidad también presenta una media mayor en las mujeres $(U=48,45 ; \mathrm{p}=.001)$, con un efecto pequeño $(r=.13)$. Se repite esta misma tendencia en el esquema de apego inmaduro $(U=46,27 ; p=.01)$, en el que las mujeres puntúan por encima de los hombres con un tamaño efecto pequeño, aunque cercano a medio $(r=.20)$. Del mismo modo, en el esquema de autosacrificio se aprecia la misma tendencia para las mujeres $(U=48,45 ; \mathrm{p}=.001)$ respecto a los hombres con un efecto pequeño $(r=.13)$. Por el contrario, los varones tienen tenuemente más activado el esquema de insuficiente autocontrol que las mujeres $(U=34,95 ; p=.018)$, si bien el tamaño efecto es prácticamente inexistente $(r=.09)$.

Con relación a la exploración de diferencias en los esquemas por formación de Grado cursada (prueba de Kruskal-Wallis para k muestras independientes y posteriores comparaciones 2 a 2 con $U$ de Mann Whitney), encontramos pocas diferencias estadísticamente significativas y, cuando existieron, presentaron un pequeño tamaño efecto.

En este sentido, tan sólo en el esquema de privación emocional existieron diferencias entre el grado en Educación Infantil y el Grado de Educación Social $(U=62,04 ; p=.037)$, con puntuaciones medias superiores en este último, aunque con un pequeño efecto $(r=.10)$.

En definitiva, podemos señalar que no encontramos grandes diferencias en los esquemas desadaptativos evaluados en virtud ni de la titulación cursada ni del género de los estudiantes.

Tabla 2

Diferencias de género estadísticamente significativas ( $U$ de Mann-Whitney, a=.05)

\begin{tabular}{llllcll}
\hline \multicolumn{1}{c}{ Esquema } & Género & $\mathbf{n}$ & Rango & $\mathbf{U}$ & $\mathbf{p}$ & $\mathbf{r}$ \\
\hline Abandono & Mujeres & 570 & 366,18 & 45,95 & .01 & .09 \\
& Hombres & 143 & 320,42 & & & \\
Vulnerabilidad & Mujeres & 570 & 370,51 & 48,45 & .01 & .13 \\
& Hombres & 143 & 303,15 & & & \\
Apego inmaduro & Mujeres & 570 & 366,69 & 46,27 & .01 & .20 \\
& Hombres & 143 & 318,37 & & & \\
Autosacrificio & Mujeres & 570 & 370,54 & 48,45 & .01 & .13 \\
& Hombres & 143 & 303,01 & & & \\
Insuficiente & Mujeres & 570 & 346,83 & 34,95 & .01 & .09 \\
Autocontrol & Hombres & 143 & 397,55 & & & \\
\hline
\end{tabular}

Fuente: Elaboración propia 
Por otra parte, en lo relativo a las posibles diferencias en las dimensiones de Inteligencia Emocional Percibida, podemos ver en la Tabla 3 que la variable género de los estudiantes mostró claras diferencias en 2 de los 3 factores de Inteligencia Emocional sometidos a medida mediante el TMMS-24 a partir de los contrastes realizados con la prueba t de Student para muestras independientes.

Tabla 3

Diferencias de medias en función de las variables género ( $t$ de Student)

\begin{tabular}{lccccc}
\hline Género & $\begin{array}{c}\text { Hombres }(\mathrm{n}=143) \\
\text { Media (DT) }\end{array}$ & $\begin{array}{c}\text { Mujeres }(\mathrm{n}=570) \\
\text { Media (DT) }\end{array}$ & $\boldsymbol{t}_{(713)}$ & $\boldsymbol{P}$ & Efecto $_{(\mathrm{d})}$ \\
\hline Atención & $25,48(6,34)$ & $27,66(6,06)$ & -3.796 & $.000^{*}$ & .35 \\
Claridad & $26,43(6,33)$ & $25,36(6,37)$ & 1.800 & .072 & .16 \\
Regulación & $27,59(6,37)$ & $26,31(6,27)$ & 1.800 & $.031^{*}$ & .20 \\
\hline
\end{tabular}

Fuente: Elaboración propia

En concreto, resultó que las mujeres prestan mayor atención a los sentimientos (media= $27,66)$ que los varones (media $=25,48)\left(t_{713}=-3.796, p=.000\right)$, con un tamaño efecto $d=.35 * *$. También existieron diferencias estadísticamente significativas en la dimensión regulación del TMMS $24\left(t_{713}=1.800, p=.031\right)$ siendo en esta ocasión la media en los hombres más alta que en las mujeres ( $M=26,31 \mathrm{vs} H=27,59)$, con un tamaño efecto $d=.20^{*}$.

Respecto al tipo de grado no se encontraron diferencias significativas en estas dimensiones.

\section{3.-Explorar la posible asociación entre Inteligencia Emocional Percibida (IEP) y los Esquemas Desadaptativos Tempranos}

Respecto a la relación entre la Inteligencia Emocional Percibida y los Esquemas Desadaptativos Tempranos, la Tabla 4 muestra que se encontraron numerosas correlaciones estadísticamente significativas (prueba chi cuadrado de Pearson) entre los 3 factores de IEP y los quince esquemas desadaptativos evaluados.

Tabla 4

Correlaciones bivariadas ( $r$ de Pearson) entre inteligencia emocional y esquemas desadaptativos

\begin{tabular}{|c|c|c|c|c|c|c|c|c|c|c|c|c|c|c|c|}
\hline & $\mathrm{Pe}$ & $A b$ & $\mathrm{De}$ & $\mathrm{Ai}$ & Im & $\mathrm{Fr}$ & Dp & $V p$ & Ap & Su & $\mathrm{Au}$ & le & Mi & $G r$ & la \\
\hline Atención & .040 & $.249^{* *}$ & $.148^{* *}$ & $.080 *$ & -.014 & .016 & $.112^{* *}$ & $.213^{* *}$ & $.186 *$ & $.109^{* *}$ & $.185^{* *}$ & $-.152^{* *}$ & $.120^{* *}$ & $.163^{* *}$ & $.086^{*}$ \\
\hline Claridad & $.092^{* *}$ & -.062 & $-.157^{* *}$ & $-.09^{* *}$ & $-.195^{* *}$ & $-.145^{* *}$ & $-.129^{* *}$ & $-.090^{*}$ & .008 & $-.187^{* *}$ & .050 & $-.189^{*}$ & $.090^{*}$ & .023 & $-.134^{* *}$ \\
\hline Reparación & $-.186^{*}$ & $-.105^{* *}$ & $-.185^{* *}$ & $-.214^{* *}$ & $-.299^{* *}$ & $-.187^{* *}$ & $-.172^{* *}$ & $.098^{* *}$ & -.053 & $-.198^{* *}$ & .060 & $-.161^{* *}$ & .061 & .021 & $-.104^{* *}$ \\
\hline TOTAL & $.568^{* *}$ & $.632^{* *}$ & $.676^{* *}$ & $.603^{* *}$ & $.671^{* *}$ & $.596^{* *}$ & $.580^{* *}$ & $.572^{* *}$ & $.517^{* *}$ & $.687^{* *}$ & $.473^{* *}$ & $.501^{* *}$ & $.345^{* *}$ & $.424^{* *}$ & $.570^{* *}$ \\
\hline
\end{tabular}

Fuente: Elaboración propia

Los esquemas que más se relacionan con la atención a los sentimientos son el de abandono y el de vulnerabilidad al peligro $\left(.249^{* *}\right.$ y $\left..213^{* *}\right)$ respectivamente lo hacen de forma positiva. 
Los otros dos factores de inteligencia emocional (Claridad emocional y Autorregulación emocional) correlacionan de forma negativa con casi todos los esquemas (tabla 4).

A continuación, se exponen las correlaciones de los factores de inteligencia emocional y dimensiones o factores que agrupan diferentes esquemas desadaptativos (tabla 5).

Tabla 5

Correlaciones inteligencia emocional y dimensiones de esquemas

\begin{tabular}{|c|c|c|c|c|c|c|c|c|}
\hline TOTAL & TOTES & F1 & $\mathrm{F}_{2}$ & $\mathrm{~F}_{3}$ & $\mathrm{~F}_{4}$ & $\mathrm{~F}_{5}$ & Atención & Claridad E \\
\hline $\begin{array}{l}\text { Factor1.Desconexión y } \\
\text { rechazo }\end{array}$ & $.809^{* *}$ & & & & & & & \\
\hline $\begin{array}{l}\text { Factor2. Desempeño y } \\
\text { autonomía deficientes }\end{array}$ & $.797^{* *}$ & $.531^{* *}$ & & & & & & \\
\hline Factor3.Límites insuficientes & $.616^{* *}$ & $.369^{* *}$ & $.426^{* *}$ & & & & & \\
\hline Factor4.Tendencia al otro & $.700^{* *}$ & $.442^{* *}$ & $.492^{* *}$ & $.292^{* *}$ & & & & \\
\hline $\begin{array}{l}\text { Factor5. Sobrevigilancia e } \\
\text { inhibición }\end{array}$ & $.567^{* *}$ & $.383^{* *}$ & $.235^{* *}$ & $.251^{* *}$ & $.368^{* *}$ & & & \\
\hline Atención & $.191^{* *}$ & $.086^{*}$ & $.194^{* *}$ & $.154^{* *}$ & $.191^{* *}$ & -.028 & & \\
\hline Claridad & $-.144^{* *}$ & $-.168^{* *}$ & $-.120^{* *}$ & -.068 & -.059 & -.073 & $.208^{* *}$ & \\
\hline Regulación & $-.199^{* *}$ & $-.277^{* *}$ & $-.172^{* *}$ & -.051 & -.058 & -.072 & $.076^{*}$ & $.328^{* *}$ \\
\hline
\end{tabular}

Se comprueba que la atención emocional vuelve a relacionarse de forma positiva con casi todos los factores, y claridad emocional y regulación emocional lo harían de forma negativa con los factores 1 (Desconexión y rechazo) y factor 2 (Desempeño y autonomía deficientes) (Tabla 5).

\section{Discusión y conclusiones}

Se ha obtenido evidencia sobre la relación existente entre los constructos Inteligencia Emocional Percibida y los Esquemas Desadaptativos Tempranos en estudiantes de educación por primera vez. Estos resultados vienen a apoyar el hallazgo de Tianguan y Joanna (2020) sobre la relación inversa entre inteligencia emocional y esquemas desadaptativos tempranos con población general. Estos mismos autores refieren que las dificultades en la regulación emocional dispararían más fácilmente los esquemas desadaptativos tempranos. Se ha constatado, por otro lado, como habitual en la perpetuación de dichos esquemas la tendencia a utilizar estrategias de evitación ante situaciones estresantes, lo que restaría oportunidades para regular emociones negativas intensas. Todo ello se relacionaría, a su vez, con una cierta incapacidad para la comprensión de las emociones propias, tal y como sugiere nuestro estudio.

La relación directa encontrada entre el componente atención a los sentimientos y Esquemas Desadaptativos Tempranos es coherente con la literatura actual. De algún modo, las heridas emocionales tempranas vinculadas a cuidadores negligentes o que generan esquemas de abandono emocional podrían sembrar, en muchos casos, una actitud de excesiva atención al propio campo emocional como señal de alerta (Ahmadpanah, 2017). En esta línea, nuestros resultados apuntan a que los esquemas de abandono y vulnerabilidad al peligro son los que se asocian en mayor medida con atención a los sentimientos. Como sabemos, no resulta 
adecuada una pobre atención emocional pero tampoco si es excesiva (García Domingo, 2021). En cualquier caso, los valores medios de nuestra muestra en las tres dimensiones de inteligencia emocional se sitúan dentro de la adecuación establecida por el propio instrumento de medida.

Sin embargo, las mujeres de nuestro estudio obtienen puntuaciones más altas en atención a los sentimientos que los hombres, y estos a su vez superiores en regulación emocional. Sobre la base de los tres dominios de inteligencia emocional, nuestros resultados coinciden parcial o totalmente con investigaciones previas donde existen alguna diferencia en alguna de las dimensiones para futuros educadores Jiménez-Hernández et al., (2019), Carretero y Nolasco, (2016), respecto a las diferencias de género. No son coincidentes con otros estudios OrtegaÁlvarez et al., (2015), Cazalla-Luna y Molero (2016), Pérez-Bonet y Velado (2017), por lo que sigue siendo un asunto controvertido y sin determinar. No obstante, conviene recordar que los estudios sobre diferencias de género en Inteligencia Emocional a través de escalas auto informadas contrastan con las realizadas a través de escalas de ejecución, reflejando que los hombres sobrevaloran sus habilidades emocionales (Subverviola 2020).

Respecto a los esquemas más prevalentes en la población universitaria de Grados en Educación estudiada son por este orden: autosacrificio, metas inalcanzables y abandono. Los tres esquemas son los más activos tanto en mujeres como en varones y coinciden, mayoritariamente, como los más prevalentes con otras muestras de estudiantes universitarios españoles (Calvete, 2005, 2020), si bien en nuestra muestra aparecen puntuaciones más altas que en las referidas. Por tanto, queda por confirmar que estos tres esquemas sean especialmente altos en los futuros educadores e incluso en educadores en ejercicio.

Respecto a las diferencias por género en base a los distintos esquemas, podemos comprobar como las mujeres presentan puntuaciones más altas en 12 de los 15 esquemas, resultado en línea con muestras similares (Calvete, 2020). No obstante, diferencias estadísticamente significativas solo las encontramos en los esquemas de abandono, vulnerabilidad, apego inmaduro y autosacrificio a favor de las mujeres y en el esquema de insuficiente autocontrol estarían los hombres por encima de las mujeres. En todos los casos los tamaños de efecto son pequeños por lo que podemos sugerir que no existen diferencias notables. No obstante, estos hallazgos coinciden mayoritariamente con otros estudios donde las mujeres parecen reflejar parte de los estereotipos de género basados en la búsqueda de compañía, el autosacrificio vinculado a la crianza y la necesidad de protección (Diana et al, 2019). Parece, por otro lado congruente, que en profesiones de ayuda a otros, como sería la de educadores, exista una tendencia a poner en el centro a los demás, en este caso en los alumnos y alumnas. Pero el descuido de las propias necesidades emocionales junto a la interiorización de ciertos estereotipos de género podrían poner en un mayor riesgo a las futuras educadoras, con relación al burnout (Qaderijam et al. 2015), a la ansiedad y la depresión.

En cualquier caso, tanto futuros educadores como educadoras pueden mejorar su salud global desde un abordaje conjunto de desactivación de esquemas desadaptativos y activación de inteligencia emocional de cara a la mejora de sus propias competencias emocionales y a la prevención de desajustes emocionales, como se ha sugerido a partir de algunas intervenciones grupales (Morvaridi et al, 2019).

Entre las limitaciones del presente estudio destacamos que la muestra utilizada se circunscribe esencialmente a un centro universitario de carácter privado en la región de Madrid, por lo que es necesario ampliar y distribuir la muestra a todos los cursos de los grados educativos y regiones españolas, de cara a confirmar los esquemas más salientes en futuros educadores y si existen cambios a lo largo de dichos estudios. Por otro lado, la medición de 
la inteligencia emocional a través de autoinforme puede generar sesgo en los resultados, así como en la propia autoevaluación de los Esquemas Desadaptativos Tempranos. Como líneas de futuro, se tendrán que intentar explorar las relaciones de estos esquemas desadaptativos con escalas de inteligencia emocional de ejecución para comprobar si se repiten las tendencias antes mencionadas. Se tendrá que continuar indagando en posibles diferencias de género que puedan suponer un mayor riesgo para la salud de las futuras docentes.

En cualquier caso, conocer estos resultados puede generar un punto de partida para los especialistas de áreas de la educación y psicología en la elaboración de programas de prevención e intervención que combinen esencialmente las dimensiones de claridad y regulación emocional con la reducción de los esquemas de autosacrificio, metas inalcanzables y abandono. Las propuestas de formaciones grupales para desactivar esquemas desadaptativos y activar inteligencia emocional puede ser una línea formativa transversal en la formación inicial y permanente de los educadores, dado el potencial valor protector de ambos en la prevención de cuadros inhabilitantes desde el punto de vista socioemocional.

\section{Referencias}

Ahmadpanah, M., Astinsadaf, S., Akhondi, A., Haghighi, M., Bahmani, D. S., Nazaribadie, M., y Brand, S. (2017). Early maladaptive schemas of emotional deprivation, social isolation, shame and abandonment are related to a history of suicide attempts among patients with major depressive disorders. Comprehensive psychiatry, 77, 7179. https://doi.org/ 10.1016/j.comppsych.2017.05.008

Antoñanzas, J. L., Salavera, C., Teruel, P., Sisamon, C., Ginto, A., Anaya, A. y Barcelona, D. (2014). Emotional intelligence and personality in student teachers. Procedia, Social and Behavioral Sciences, 132, 492-496. https://doi.org/10.1016/j.sbspro.2014.04.342

Ato, M., López, J.J. y Benavente, A. (2013). Un sistema de clasificación de los diseños de investigación en psicología. Anales de Psicología, 29(3), 1038-1059.

.Bilge, Y. y Balaban, G. (2021): The relationship between Personality disorders and Early Maladaptative Schemas and the moderating role of gender.Alpha Psychiatry,22(1),1218. https://doi.org/ 10.5455/apd.114935

Bisquerra,R., Pérez J.C., y García,E. (2015).Inteligencia emocional en educación. Síntesis.

Bisquerra, R. (2016). 10 ideas clave. Educación emocional. Graó.

Calvete, E., Estévez, A., López de Arroyabe, E., y Ruiz, P. (2005). The Schema QuestionnaireShort form: Structure and relationship with automatic thoughts and symptoms of affective disorders. European Journal of Psychological Assessment, 21, 91-100. https://doi.org/10.1027/1015-5759.21.2.90

Calvete, E., González Díez, Z. y Orúe, I., (2013). An examination of the Structure and Stability of Early Maladaptive Schemas by means of the Young Schema Questionnaire-3. European Journal of Psychological Assessment, 29(4), 283-290. https://doi.org/10.1027/1015-5759/a000158 
Calvete, E. (2020). Cuestionario de Esquemas de Young: Adaptación de una versión breve para adolescentes y jóvenes españoles. Revista de Psicopatologia y Psicologia Clinica, 25(3), 219-229. https://doi.org/10.5944/rppc.29013

Carretero, R. y Nolasco, A. (2016). La inteligencia emocional desde la perspectiva sexista. En F. Soler., L. Aparicio., L.O. Díaz, E. Escolano y A. Rodríguez. (Eds.), Inteligencia emocional y bienestar, (pp .445-454). Universidad San Jorge.

Cazalla-Luna, N. y Molero, D. (2016). Inteligencia emocional percibida, disposición al optimismo-pesimismo, satisfacción vital y personalidad de docentes en su formación inicial. Revista de Investigación Educativa, 34(1), 241-258. http://dx.doi.org/10.6018/rie.34.1.220701

Cid, J., Tejero, A., y Torrubia, R. (1997). Adaptación española del cuestionario de Esquemas de Young. [Spanish version of the Young Schema Questionaire]

Diana L. Janson, Craig A. Harms, Ross C. Hollett y Robert D. Segal (2019). Differences between Men and Women Regarding Early Maladaptive Schemas in an Australian Adult Alcohol Dependent Clinical Sample, Substance Use \& Misuse. https://10.1080/10826084.2018.1480038

Dolev, N. y Leshem, S. (2017). Developing emotional intelligence competence among teachers. Teacher development, 21-39. https://doi.org/10.1080/13664530.2016.1207093

Dominguez-Lara, S. (2018). Influencia de la inteligencia emocional y personalidad en las estrategias cognitivas de regulación emocional en la desaprobación de exámenes en estudiantes de psicología. Cultura: Revista de la Asociación de Docentes de la USMP, 32. 225-259.

Fernández-Berrocal, P., Extremera, N. y Ramos, N. (2004). Validity and reliability of the Spanish modified version of the Trait Meta-Mood Scale. Psychological Report, 94, 751755 .

Fernández-Berrocal, P., Cabello, R. y Gutiérrez-Cobo, M.J. (2017). Avances en la investigación sobre competencias emocionales en educación. Revista Interuniversitaria de Formación del Profesorado, 88, 15-26.

García-Domingo, B. (2021). Competencia emocional en maestros de educación infantil y primaria: fuentes de variabilidad y sugerencias de mejora. Revista Electrónica Interuniversitaria de Formación del Profesorado, 24(2), 1-15. http://dx.doi.org/10.6018/reifop.450111

Gholami, A., Ghanavati, My. \& Suleiman, M. (2013). Determining the relationship between Early Maladaptive Schemas and Isfahan City's Physical Education Teachers Happiness. International Journal of Basic Sciences \& Applied Research, (3), 167-169.

Janovsky T., Rock A.J., Thorsteinsson E.B., Clark G.I., Murray C.V. (2020). The relationship between early maladaptive schemas and interpersonal problems: A meta-analytic review. Clin Psychol Psychother.1-40. https://doi.org/10.1002/cpp.2439 
Jiménez Hernández, D., Sancho Requena, P., Sánchez Fuentes, S. (2019). Perfil del futuro docente: Nuevos retos en el marco del EES. Contextos Educativos 23, 125-139. DOI: http://dx.doi.org/10.18172/con.3471

Kotsou, I., Mikolajczak, M., Heeren, A., Grégoire, J., y Leys, C. (2019). Improving emotional intelligence: A systematic review of existing work and future challenges. Emotion Review, 11(2), 151-165. https://doi.org/10.1177\%2F1754073917735902

Livia, G. y García, L. (2015). Estudio de las competencias socio-emocionales y su relación con el afrontamiento en futuros profesores de nivel medio. REIFOP. Revista Electrónica Interuniversitaria de Formación del Profesorado, 18(1), 213-228. https://doi.org/10.6018/reifop.18.1.193041

Malahat, M. (2018). The moderating role of lifestyleon maladaptatives Schemas and responsability among iranian male teachers. International Journal of Applied Behavioural Sciences, 5(2), 44-51. https://doi.org/10.22037/ijabs.v5i2.21814

Márquez-Cervantes, M.C. \& Gaeta-González, M.L. (2017). Desarrollo de competencias emocionales en pre-adolescentes: el papel de padres de docentes. Revista Electrónica Interuniversitaria de Formación del Profesorado, 20(2), 221-235. https://doi.org/10.6018/reifop/20.2.232941

Mayer, J.D. \& Salovey, P. (1997). What is emotional intelligence? En P. Salovey \& D. Sluyter (Eds.). Emotional Development and Emotional Intelligence: Implications for Educators (pp. 3-31). Basic Books

Morvaridi, M., Mashhadi, A., Shamloo, Z. S., \& Leahy, R. L. (2019). The effectiveness of group emotional schema therapy on emotional regulation and social anxiety symptoms. International Journal of Cognitive Therapy, 12(1), 16-24.

https://doi.org/10.4103\%2Fabr.abr_113_16

Ortega-Navas, M. C. (2014). La mejora de la empleabilidad en los estudiantes de la educación superior: el uso de la inteligencia emocional como estrategia educativa. Procedia Social and Behavioral Sciences, 139, 380-386. https://doi.org/10.1016/j.sbspro.2014.08.021

Ortega-Álvarez, F., Núñez-Hergueta, E., Molero, D. y Torres-González, J. A. (2015). Diversidad emocional y satisfacción vital en futuros docentes. Revista de Educación Inclusiva, 8(3), 205-217.

Pena, M., Rey, L. y Extremera, N. (2012). Life Satisfaction and Engagement in Elementary and Primary Educators: Differences in Emotional Intelligence and Gender. Revista de Psicodidáctica, 17(2), 341-358. https://doi.org/10.1387/Rev.Psicodidact.1220

Pérez Bonet, G. y Velado Guillén, L. A. (2017). Inteligencia Emocional Percibida (IEP) en el alumnado universitario de educación. Análisis comparativo por género y grado. Escuela Abierta (EA), 20, 23-34. https://doi.org/10.29257/EA20.2017.03

Pérez-González, J. C. (2012). Revisión del aprendizaje social y emocional en el mundo. En R. Bisquerra (Coord.), ¿Cómo educar las emociones? La inteligencia emocional en la infancia y la adolescencia (pp. 56-69). Hospital Sant Joan de Déu. 
Puertas Molero, P., Ubago Jiménez, J.L., Moreno Arrebola, R., Padial Ruz, R., Martínez Martínez, A. y González Valero. G. (2018). La inteligencia emocional en la formación y desempeño docente: una revisión sistemática. Revista Española de Orientación y Psicopedagogía, 29(2), 128-142. https://doi.org/10.5944/reop.vol.29.num.2.2018.23157

Qahderijani Mahboubeh, M., Homaei, R., Mehdizadeh, S. y Norouzi, M. (2015). The Relationship between Early Maladaptive Schemas and Esfahan Guidance School Teachers' Job Burnout. The International Journal of Indian Psicología, 3(6), 68-73.

Rittenmyer,G.J.(1997). The relationship between schemas and job burnout Among public school teachers. Tesis doctoral, Universidad de Pacífico. https://scholarlycommons.pacific.edu/uop_etds/2755

Saliari, A., Moslehi, M. y Valizadehi, R. (2011). Relationship between emotional intelligence and burnout syndrome in sport teachers of secondary schools. Procedia Social and Behavioural Sciences, 15, 1786-1791. https://doi.org/10.1016/j.sbspro.2011.04.003

Sanmartín López, R., Gonzálvez Maciá, C. y Vicent Juan, M. (2018). Inteligencia emocional en alumnado de formación profesional. Diferencias en función del curso, del género y de la edad. Educar, 54(1), 229-245. https://doi.org/10.5565/rev/educar.797

Suberviola Oveja, I. (2020). La socialización diferencial emocional de género como factor predictor del carácter. iQUAL. Revista de Género e Igualdad, 3, 80-93.https:// doi. 10.6018/iqual.36961

Tianyuan, K. \& Joanne, B. (2020). Thinking about feeling: Using trait emotional intelligence in understanding the associations between early maladaptatives schemas and coping styles. Psychology and Psychoterapy: Theory, Research and Practise, 93(1), 1-20. https://doi.org/10.1111/papt.12202

UNESCO (2018). Education 2030. Incheon Declaration and Framework for Action. http://uis.unesco.org/sites/default/files/documents/education-2030-incheonframework-for-action-implementation-of-sdg4-2016-en_2.pdf

Young, J., Closco, J. y Weishaar, M. (2016). Terapia de esquemas. Guía práctica. Desclée de Brouwer. 